\title{
Zusammensetzung des Kolostrums bei einigen Fleischrinderrassen in Abhängigkeit von der nach dem Abkalben vergangenen Zeit
}

\author{
Herrn Professor Dr. Dr. h.c. Gottfried Leuthold zum 65. Geburtstag gewidmet
}

\section{Summary}

Title of the paper: The composition of the colostrum of some beef cattle breeds according to time of post partum

This research was carried out to investigate the changes of main components of colostrum with 0-4 days after calving by five beef cattle breds, kept in Hungary.

After calving and 24 hours later beef cattle breeds had higher dry matter content in colostrum compared to that of dairy breeds; but the dairy breeds were superior to the beef cattle breeds from the second day. This relates to the colostrum of the beef cattle breeds more concentrated than the dairy breeds during the first 24 hours but they reached that values earlier which were characteristic to the normal milk. Examining the protein content of colostrum there were similar tendencies in change of dry matter content with difference that the protein content of colostrum of beef cattle breeds was higher than that of the dairy breeds between the $2^{\text {nd }}$ and $4^{\text {th }}$ days, too. On the first and the second days the fat content of colostrum of the sucklers decreased which was related to the higher dilution in beef cattle breeds. The sugar content of colostrum of the beef cattle breeds has increased between the calving and the $4^{\text {th }}$ day post partum, and reached the values characteristic to the normal milk - (4.8 $5.0 \%)$ - on the $5^{\text {th }}-6^{\text {th }}$ day. There was not significant difference between the beef and the dairy breeds, neither in sugar nor in the ash content. The ash content of colostrum reached the values characteristic to the normal milk, 7-8 days after calving.

The whey protein and the non protein nitrogen content of colostrum of the beef cattle breeds were higher than that of the dairy breeds, significantly. Decreasing of these fractions in the beef cattle breeds were more moderate than the dairy breeds as in case of the total protein content, according to time. Our calculations show that whey protein content of the beef cattle breeds reached that values which were characteristic to the normal milk, after the $5^{\text {th }}$ day post partum. Not having found a decided difference in the casein content of colostrum between the beef and the dairy breeds. The casein content of colostrum of blonde d' Aquitaine and Limousin higher than that of the other kind of beef cattle breeds, which manifests itself in the ratio of whey protein and casein, too. At the same time the ratio of whey protein and casein in both Angus types differ from the other beef cattle breeds in favour of the whey protein.

The calculated immunglobulin-G values of colostrum milked immediately after calving were usually much higher at sucklers than at the dairy breeds. This was true especially between the $1^{\text {st }}$ and the $4^{\text {th }}$ days which was connected with the more moderate decrease of $\mathrm{IgG}$ fraction in the beef cattle breeds. Similarly the data of the literature the IgG content of the fresh milked colostrum was determined primarily by the breed. According to our examinations this determination becomes more unimportant moving off the time of calving.

The energy content of colostrum at Black Angus and Red Angus rose among the other kind of beef cattle breeds. The average energy content of the colostrum of blonde d' Aquitaine and Limousin was connected with the high protein content of their colostrum. The energy content of colostrum of Hungarian Simmental was the lowest.

Key words: colostrum, immunglobulin-G, component of colostrum, beef cattle, energy content

\section{Zusammenfassung}

Untersucht wurden die Veränderungen in der Zusammensetzung des Kolostrums in den ersten vier Tagen nach dem Abkalben bei fünf in Ungarn gehaltenen Fleischrinderrassen. 
Der Trockensubstanzgehalt im Kolostrum ist unmittelbar nach der Abkalbung sowie am ersten Tag danach bei Fleischrinderrassen höher als bei Milchrindern; ab dem zweiten Tag dagegen ist dieses Verhältnis umgekehrt. Das deutet darauf hin, daß das Kolostrum der Fleischrassen zu Beginn zwar konzentrierter ist, aber zeitmäßig eher den Trockensubstanzgehalt der Vollmilch erreicht. Ähnlich ist die Situation beim Eiweißgehalt, nur daß er bei den Fleischrassen nicht nur in den ersten 48 Stunden, sondern auch noch am 2.-4. Tag nach dem Abkalben höher liegt als bei Milchkühen. Bei allen Fleischrassen sinkt der Fettgehalt des Kolostrums am ersten und 2. Tag in hohem Maße ab, was auf eine größere Verdünnung schließen läßt. Der Zuckergehalt dagegen erhöht sich ständig vom Abkalbezeitpunkt an bis zum 4. Tag danach und erreicht erwartungsgemäß am 5.-6. Tag den für Vollmilch charakteristischen Wert von 4,8-5,0\%. Hinsichtlich des Zuckergehaltes gibt es zwischen den Nutzungstypen keinen Unterschied, was auch auf den Aschegehalt zutrifft, der etwa am 7.-8. Tag den normalen Vollmilchwert erreicht.

Im Kolostrum der Fleischrassen ist ein bedeutend höherer Anteil an Molkeneiweiß bzw. NPN zu finden, als bei den Milchrassen. Mit wachsendem Zeitabstand von der Abkalbung ist auch bei diesen Fraktionen - ebenso wie beim Gesamteiweißgehalt - ein geringeres Absinken zu beobachten. Den vorliegenden Untersuchungen zufolge erreicht der Molkeneiweißgehalt des Kolostrums bei den Fleischrassen erst am 5. Tag den Vollmilchwert. Hinsichtlich des Kaseingehaltes gibt es zwischen den beiden Nutzungstypen keinen sonderlich großen Unterschied. Nur bei den Rassen Blonde d'Aquitaine und Limousin ist er höher als derjenige der anderen Rassen, was sich auch im Molkeneiweiß-Kasein-Verhältnis zeigt. Gleichzeitig weicht das Molkeneiweiß-Kasein-Verhältnis beider Angusrassen von dem der anderen Fleischrassen zugunsten des Anteils an Molkeneiweiß ab.

Im Kolostrum der Fleischkühe übersteigen die errechneten Immunglobulin-G-Werte (Immunglobulin G, IgG) im allgemeinen diejenigen der Milchrassen, besonders am 1.-4. Tag, was mit geringeren Veränderungem des Immunglobulin-G-Gehaltes bei den Fleischrinderrassen gegenüber den Milchrassen zusammenhängt. In der Literatur wird hinsichtlich des IgG-Gehaltes im erstgemolkenen Kolostrum übereinstimmend die Abhängigkeit von der Rasse betont. In vorliegenden Untersuchungen werden diese Unterschiede mit wachsendem Zeitabstand vom Kalben an unwesentlich.

Der Energiegehalt ist besonders bei den Schwarze Angus und den Red Angus gegenüber den anderen Fleischrassen hervorzuheben. Der Energiegehalt bei den Blonde d'Aquitaine und Limousin ist mittelmäßig, was dem hohen Eiweißgehalt des Kolostrums zu verdanken ist. Der Energiegehalt im Kolostrum des Ungarischen Fleckviehs ist am niedrigsten.

Schliusselwörter: Kolostrum, Immunglobulin-G, Milchinhaltsstoffe, Fleischrinderrassen, Energiegehalt

\section{Einleitung}

Das wichtigste Produkt in der Fleischrinderhaltung ist das entwöhnte Kalb. Deshalb ist es sehr wichtig, daß Mutterkühe regelmäßig besamt werden und einmal jährlich kalben.

Das geborene Kalb allein ist dagegen nur wenig wert, denn trotz der anfänglichen Immunität und einer gesunden Haltung können zahlreiche Einflüsse bis zur Entwöhnung seinen Tod verursachen. Aus der Praxis ist bekannt, daß die Verluste bis zum Absetzen sogar $10 \%$ betragen können. Die Verluste sind - ausgenommen die angeborenen und erworbenen Krankheiten - grundsätzlich auf Haltungs- bzw. Fütterungsfehler zurückzuführen. Die Umweltfaktoren der Haltungsumstände wurden von vielen Autoren untersucht, über den Zusammenhang zwischen Technologie und Kälberverlusten gibt es ebenfalls eine Reihe von Publikationen. In der Praxis existieren viele unterschiedliche Lösungen für die Aufzucht säugender Kälber, um Verluste zu verringern.

Die einzige Nahrung des Kalbes nach der Geburt ist das Kolostrum. Seine Menge, Energiegehalt und Zusammensetzung werden durch Umweltfaktoren bestimmt, die höchstens mittelbar über die Fütterung der Mutterkuh beeinflußt werden können, was für eine gesunde Kälberaufzucht zumindest ebenso wichtig ist, wie die Unterbringung oder die Haltung. 
Da die Komponenten im Kolostrum von Rinderrassen bisher nur wenig untersucht wurden, und bei Fleischrassen kaum Daten zur Verfügung stehen, war es das Ziel dieser Arbeit, das Kolostrum einiger in Ungarn gehaltener Fleischrassen im Hinblick auf die Zusammensetzung des Kolostrums in Abhängigkeit von der nach dem Abkalben vergangenen Zeit zu untersuchen.

\section{Literaturübersicht}

Über die wichtigsten Komponenten im Kolostrum liegen Literaturangaben vor, jedoch nur wenige Daten hinsichtlich der Fleischrassen. ÁLDÁSY und ERỐS (1969) stellten im Kolostrum von 26 Ungarischen Fleckviehkühen direkt nach dem Abkalben 14,2\% Eiweiß fest (min. 9,6\%, max. 19,6\%), nach 24 Stunden 7,5\% (min. 5,8\%, max. 10,8\%), nach 72 Stunden 2,5\% (min. 1,2\%, max. 3,7\%). SEBELA und KLICNIK (1975) fanden im Kolostrum von 4 Dänischen Rotkühen am ersten Tag 10,38\% Eiweiß (min. 3,55\%, max. 15,18\%), am dritten Tag 3,88\% und am 5. Tag 3,29\%. KVAPILIK et al. (1975) stellten bei 14 Milchkühen im Kolostrum des 1. Tages einen Eiweißgehalt von $14,64 \%$, einen Kaseingehalt von 6,37\% und Serumeiweiß von $8,26 \%$ fest. Diese Werte sanken innerhalb von 3 Tagen auf 4,59 - 3,20 - 1,34\%, nach 5 Tagen auf 4,16 - 3,15 $1,01 \% \mathrm{ab}$.

Laut MERÉNYI et al. (1996) ist der Gehalt an Molkeneiweiß und Kasein im Kolostrum deshalb so hoch, weil dies die wichtigste Eiweißquelle für das Kalb darstellt, während gleichzeitig der Milchzuckergehalt niedrig ist. Ein hoher Gehalt an Molkeneiweiß ist auch deshalb sehr wichtig, da sein biologischer Wert um das Eineinhalbfache höher ist, als der des Kaseins - (CSAPÓ et al., 1988) - und diese Fraktion auch die Immunglobuline enthält (CSAPÓ et al., 1991, 1994). In der Fleischrinderhaltung ist ein hoher biologischer Wert der Muttermilch von besonderer Bedeutung, da sie die einzige Nahrung für das Kalb darstellt (KOVÁCS, 1997b).

Tabelle 1

Komponenten im Kolostrum von Milchrassen zu verschiedenen Zeitpunkten nach dem Abkalben (Some components of dairy breeds' colostrum in different time after calving)

\begin{tabular}{|c|c|c|c|c|c|c|c|c|c|c|}
\hline \multirow{2}{*}{$\begin{array}{l}\text { Rasse } \\
\text { Kompo- } \\
\text { nente }\end{array}$} & \multicolumn{5}{|c|}{ Vater: Holstein-Friesian } & \multicolumn{5}{|c|}{ Vater: Jersey } \\
\hline & 0. & 1. & 2. & 3. & 5. & 0. & 1. & 2. & 3. & 5. \\
\hline $\begin{array}{l}\text { Trocken- } \\
\text { substanz } \\
\text { Gesamt- }\end{array}$ & 25,20 & 13,45 & 13,50 & 13,42 & 12,72 & 24,41 & 14,36 & 14,50 & 14,82 & 14,9 \\
\hline $\begin{array}{l}\text { eiweiß } \\
\text { Molken- }\end{array}$ & 15,40 & 5,52 & 4,40 & 4,11 & 3,98 & 14,47 & 6,48 & 5,91 & 4,72 & 4,68 \\
\hline $\begin{array}{l}\text { eiweiß } \\
\text { Immun- }\end{array}$ & 10,12 & 1,73 & 1,35 & 1,04 & 0,80 & 10,02 & 1,67 & 1,23 & 0,89 & 0,91 \\
\hline globulin-G & 120,2 & 31,70 & 6,18 & 6,20 & 2,31 & 102,4 & 30,20 & 5,22 & 5,01 & 2,10 \\
\hline Kasein & 5,28 & 3,79 & 3,06 & 3,07 & 3,18 & 4,45 & 4,81 & 4,68 & 3,83 & 3,77 \\
\hline NPN x 6,38 & 0,43 & 0,33 & 0,27 & 0,27 & 0,20 & 0,43 & 0,38 & 0,29 & 0,28 & 0,19 \\
\hline Asche & 1,157 & 0,926 & 0,889 & 0,854 & 0,826 & 1,119 & 1,112 & 1,014 & 0,972 & 0,88 \\
\hline
\end{tabular}


CSAPÓ und CSAPÓNÉ (1984) sowie SZENTPÉTERI et al. (1986) untersuchten das Kolostrum von Vätern des Genotyps Holstein-Friesian bzw. Jersey in Abhängigkeit von der nach dem Abkalben verstrichenen Zeit hinsichtlich des Gehaltes an Trockensubstanz, Gesamteiweiß, Molkeneiweiß, Kasein, NPN und Asche (Tab. 1).

KLIMMER und SCHÖNBERG (1951) fanden einen Aschegehalt von 1,2\% im erstgemolkenen Kolostrum, der 12 Stunden nach der Abkalbung auf 1,6\% anstieg und bereits am 3. Tag den für Vollmilch charakteristischen Wert von $0,8 \%$ erreichte. SEBELA und KLICNIK (1975) untersuchten Kolostrum und Milch von 4 Dänischen Rotkühen bis zum 14. Tag nach der Abkalbung. Der Aschegehalt sank von 1,18\% am ersten Tag (min. 0,79\%, max. 1,43\%) auf 0,91\% am zweiten Tag, und nur am 13.-14. Laktationstag erreichte er den charakteristischen Vollmilchwert von 0,74-0,76\%. KIRCHMEIER (1976) fand im erstgemolkenen Kolostrum einen Aschegehalt von 1,4\% , der bereits am Ende des 1. Tages auf 1,0\% absank und am 7.-10. Laktationstag den charakteristischen Vollmilchwert von $0,8 \%$ erreichte. KOVÁCS (1997a) erhielt bei vier Fleischrassen einen durchschnittlichen Aschegehalt von 0,75 in der Vollmilch. Daten von CSAPÓ et al. (1991) sowie CSAPÓ und CSAPÓ-KISS (1994) zeigen eine enge positive Korrelation zwischen dem Molkeneiweiß und dem Immunglobulin des Kolostrums ( $r=0,97)$. Laut MIELKE (1997) hängt der Immunglobulingehalt im erstgemolkenen Kolostrum in erster Linie von der Rasse und der Anzahl der Laktationen ab. ÁLDÁSY und ERỐS (1969) stellten bei Ungarischen Fleckviehkühen im erstgemolkenen Kolostrum einen Immunglobulingehalt von 7,65\% fest, der innerhalb von 24 Stunden auf $3 \%$ absank und nach 72 Stunden nur noch $0,78 \%$ betrug. MULLER und ELLINGER (1981) untersuchten den Immunglobulin-G-Gehalt (IgG) im Kolostrum der Genotypen Ayrshire, Schweizer Braune, Guernsey, Holstein-Friesian und Jersey. Bei den Jersey fanden sie den höchsten IgG-Wert von 6,65\%, bei Holstein-Friesian den niedrigsten mit 4,12\%. NORMAN und HOHENBOKEN (1981) stellten im ersten Kolstrum des Simmentaler Rindes 120,2 mg/ml fest, SZENT-IVÁNYI (1976) im Kolostrum des Ungarischen Fleckviehs 47,6\%, in der Milch 0,59\%.

Da der IgG im Kolostrum nach dem Kalben sehr schnell absinkt, ist es wichtig, daß das Neugeborene möglichst zeitig und viel von der ersten Kolostralmilch trinkt. Hinzu kommt noch, daß neben dem schnellen Absinken der Immunstoffe laut ÁLDÁSY und ERÕS (1969) und PORTER (1972) die Aufnahme dieser Schutzstoffe nur bis zur 25.27. Lebensstunde des Kalbes möglich ist (PORTER, 1972; LOSONCZY et al., 1979).

\section{Material und Methode}

Die untersuchten Genotypen, Zeitpunkte und Orte der Kolstrum-Probenahme sowie die Anzahl der untersuchten Kühe sind der Tabelle 2 zu entnehmen.

Die erste Probenahme erfolgte innerhalb von 3 Stunden nach dem Abkalben. Die zweite und die darauffolgenden Probenahmen fanden immer zum gleichen Zeitpunkt, jeweils 24 Stunden nach der ersten, statt. Die letzte Probenahme war am 4. Tag nach dem Abkalben. Während der Probenahme wurden die Kühe angebunden und per Hand gemolken. Nach der Euterdesinfizierung wurden bis zum Erreichen der entsprechenden 
Tabelle 2

Untersuchte Genotypen, Anzahl Kühe, Zeitpunkte und Orte der Kolostrum-Probenahme (The examined breeds, their number and the location of farms and terms of gained samples)

\begin{tabular}{lccc}
\hline Rassen & Betrieb & Zeit der Probenahme & Anzahl Kühe \\
\hline Blonde d'Aquitaine & 1 & Februar 1997 & 5 \\
Schwarze Angus & 2 & März 1997 & 5 \\
Red Angus & 2 & März 1997 & 5 \\
Ungarisches Fleckvieh & 3 & April 1997 & 5 \\
Limousin & 3 & April 1997 & 5 \\
\hline
\end{tabular}

Probemenge die einzelnen Zitzen reihum immer wieder völlig ausgemolken. Die Mindestmenge betrug $50 \mathrm{ml}$, um im Labor parallel Analysen durchführen zu können.

Die Analysen wurden im Milchlaboratorium für Leistungskontrollen in der Tierzucht in Gödöllö sowie im Chemischen Institut der Fakultät Tierproduktion der PANNON Agrarwissenschaftlichen Universität in Kaposvár vorgenommen. Die Proben für das Labor in Gödöllö wurden mit Hilfe von Microtabs-Tabletten, die „Bomopron“ enthalten, konserviert und bei $+4{ }^{\circ} \mathrm{C}$ bis zur Verarbeitung aufbewahrt. Die Aufbewahrungszeit dauerte höchstens eine Woche.

In Gödöllö wurden die wichtigsten Komponenten des Kolostrums - Fett, Eiweiß, Zukker - mit dem Analysator Combi-Foss der dänischen Firma Foss-Electric untersucht. Die Proben wurden wegen ihres hohen Trockensubstanzgehaltes - in zwei Serien - mit destilliertem Wasser verdünnt und nach Vermischung und Erwärmung verarbeitet. Die Verdünnung war wegen der empfindlichen Mikropipetten des Analysators und der kalibrierten Meßskala notwendig.

In Kaposvár erfolgte die Bestimmung des Gehaltes an Trockensubstanz, Eiweiß und Asche sowie detaillierte Untersuchungen zu den Eiweißfraktionen. Zur Bestimmung dieser Fraktionen, wie bei CSAPÓ et al. (1982a, b) beschrieben, wird das gesamte Kolostrum (N\% x 6,38 = Gesamteiweiß) mit $8000 \mathrm{U} / \mathrm{min} 10$ Minuten lang mit der Zentrifuge T 30 entfettet. Für die entfettete Flüssigkeit wurde der pH-Wert auf dem pH-Meßgerät Op 264 auf 4,55 eingestellt. Danach wurde das Kasein mittels einer 10minütigen Zentrifugierung bei $8000 \mathrm{U} / \mathrm{min}$ von der Molke getrennt. Aus der Molke (N\% x 6,38=Molkeneiweiß) wurde mit 12\%iger Trichlor-Esssigsäure das Molkeneiweiß entfernt und danach der Nitrogengehalt (Nicht-Protein-Stickstoff, NPN) bestimmt. Zieht man vom Nitrogen der Molke das NPN ab, so erhält man den Nitrogengehalt des tatsächlichen Molkeneiweißes. Zieht man vom Nitrogengehalt der Vollmilch den Nitrogengehalt der Molke ab, so erhält man den Nitrogengehalt des Kaseins. Den Nitrogengehalt der Fraktionen, multipliziert mit dem Konversionsfaktor 6,38 ergibt dessen Eiweißgehalt. Der Nitrogengehalt der verschiedenen Fraktionen und Proben wurde mit dem Schnell-Nitrogenanalysator Kjell-Foss 16200 bestimmt.

Der Gehalt an Trockensubstanz wurde durch Trocknung bis zur Massenbeständigkeit (Ung. Standard Nr. 6830, Pkt. 66) ermittelt. Der Aschegehalt der Proben wurde durch Veraschung ((Ung. Standard Nr. 3726/2, Pkt. 76) bestimmt.

Entsprechend publizierter Daten von CSAPÓ et al. (1991) sowie CSAPÓ und CSAPÓKISS (1994) wurde der Immunglobulin-G-Gehalt des Kolostrums aus dem prozentualen Anteil des Molkeneiweißes errechnet und mit der linearen Regressionsformel ge- 
schätzt. Aufgrund der 54 Veränderlichen war die geschätzte Formel: Y $=\mathrm{A}+\mathrm{B}$ x X. Der , $\mathrm{r}^{\prime \prime}$-Wert betrug 0,96673 , wobei $\mathrm{A}=-0,36742$ und $\mathrm{B}=10,07453$ betrugen.

Der Energiegehalt im Kolostrum wurde wie folgt bestimmt:

- Die prozentualen Anteile der Komponenten - Eiweiß, Fett, Zucker N-freie Extraktstoffe wurden mit dem Faktor 10 multipliziert, damit die Menge in der Maßeinheit $\mathrm{g} / \mathrm{kg}$ vorlag.

- Die Verbrennungswärme der einzelnen Komponenten beträgt nach BÍRÓ und LINDNER (1995): N-freie Extraktstoffe=17,16 kJ/g; Fett=38,94 kJ/g; Eiweiß=22,19kJ/g. Da im Organismus aus Eiweiß-Nitrogen Ammonium und Karbamid entsteht, also kein N2 (Kalorimeter), wird bei Eiweiß anstatt der Verbrennungswärme mit dem spezifischen biologischen Nutzwert gerechnet (SARUDI, 1998), der mit der Verbrennungswärme der Kohlenhydrate übereinstimmt $(17,16 \mathrm{~kJ} / \mathrm{g})$.

- Auf dieser Grundlage wird der spezifische biologische Nutzwert (Kolostrum) $=17,16 x$ Eiweiß + 38,9 x Fett + 17,16 x N-freie Extraktstoffe/1000 (MJ/kg) bestimmt, der für den Vergleich der Rassen benutzt wurde.

Die statistische Auswertung erfolgte mittels t-Tests von STUDENT. Für weitere Statistik wurde die Software 7.5 SPSS for Windows (1996) genutzt.

\section{Ergebnisse}

Die wichtigsten Komponenten des Kolostrums in Abhängigkeit von der nach dem Abkalben vergangenen Zeit

In Tabelle 3 werden die wichtigsten Komponenten des Kolostrums, in Abhängigkeit von der nach dem Abkalben vergangenen Zeit aufgegliedert, vorgestellt.

Vergleicht man diese Ergebnisse mit der Literatur, dann ist festzustellen, daß der Trokkensubstanzgehalt im Kolostrum der Limousin-Rasse unmittelbar nach dem Kalben und nach 24 Stunden dem der Milchrassen ähnelt, die von CSAPÓ und CSAPÓNÉ (1984) sowie SZENTPÉTERI et al. (1986) untersucht wurden. Die Werte beim Ungarischen Fleckvieh blieben hinter diesen zurück, während bei den anderen Fleischrinderrassen der Durchschnittswert signifikant ( $\mathrm{P} \leq 0,1 \%)$ darüber liegt. 48 Stunden nach der Abkalbung änderte sich die Situation, denn dann erhielten obige Autoren bei den Milchrassen höhere Trockensubstanzwerte als die hier untersuchten Fleischrassen, mit einer Ausnahme - den Red Angus. Daraus läßt sich ableiten, daß die Trockensubstanz im Kolostrum der Fleischrassen anfangs eine höhere Konzentration hat, aber zeitmäßig früher den Vollmilchwert erreicht. Diese Feststellung wird auch dadurch bestätigt, daß die Werte beim Ungarischen Fleckvieh - obwohl als Fleischrasse gehalten - am ehesten den für Milchrassen typischen Tendenzen entsprechen.

Für die Unterschiede im Trockensubstanzgehalt, sowohl untereinander als auch mit den von CSAPÓ und CSAPÓNÉ (1984) sowie SZENTPÉTERI et al. (1986) untersuchten Milchrassen, ist der Gesamteiweißgehalt verantwortlich, denn der korrelative Gesamtwert der 125 Proben zwischen Trockensubstanz- bzw. Eiweißgehalt beträgt $r=0,931$. Der Eiweißgehalt im Kolostrum der Fleischrassen verhält sich also ähnlich wie der Trockensubstanzgehalt, nur mit dem Unterschied, daß er nicht nur in den ersten 48 Stunden sondern auch am 2.-4. Tag nach dem Abkalben noch höher ist, als bei 
Tabelle 3

Wichtigste Komponenten im Kolostrum einiger Fleischrinderrassen zu verschiedenen Zeitpunkten nach der Abkalbung (Examination of the main components at some beef cattle breeds' colostrum in different time after calving)

\begin{tabular}{|c|c|c|c|c|c|c|c|c|c|c|c|c|c|c|c|c|c|}
\hline \multirow{2}{*}{$\begin{array}{l}\text { Kompo- } \\
\text { nente }\end{array}$} & \multirow[t]{2}{*}{ Tage } & \multicolumn{3}{|c|}{ Blonde d' Aq. } & \multicolumn{3}{|c|}{ Red Angus } & \multicolumn{3}{|c|}{ Schwarze Angus } & \multicolumn{3}{|c|}{ Ung. Fleckvieh } & \multicolumn{3}{|c|}{ Limousin } & \multirow{2}{*}{$\begin{array}{c}\text { Rassen } \\
\varnothing\end{array}$} \\
\hline & & $\min$ & & $\emptyset$ & $\min$ & $\max$ & $\varnothing$ & & $\max$ & $\varnothing$ & $\min$ & & & $\min$ & & $\varnothing$ & \\
\hline \multirow{5}{*}{$\begin{array}{l}\text { Trocken- } \\
\text { substanz }\end{array}$} & 0. & 22,8 & 33,9 & 27,0 & 25,0 & 40,3 & 33,6 & 29,1 & 40,4 & 33,7 & 19,52 & 28,2 & 23,0 & 19,5 & 33,3 & 25,5 & 28,6 \\
\hline & 1. & 11,7 & 23,7 & 16,3 & 16,3 & 20,8 & 18,4 & 13,6 & 23,9 & 16,8 & 11,5 & 13,2 & 12,4 & 11,5 & 15,8 & 14,2 & 15,6 \\
\hline & 2. & 9,90 & 14,1 & 12,0 & 11,1 & 19,7 & 15,3 & 10,9 & 12,4 & 11,6 & 9,50 & 16,3 & 11,8 & 9,70 & 15,6 & 11,8 & 12,5 \\
\hline & 3. & 10,5 & 14,0 & 12,5 & 10,3 & 15,2 & 12,9 & 10,4 & 17,4 & 13,0 & 10,3 & 14,9 & 13,3 & 9,40 & 16,6 & 12,0 & 12,7 \\
\hline & 4. & 9,90 & 12,5 & 11,5 & 10,2 & 13,4 & 11,5 & 10,8 & 13,4 & 11,8 & 9,40 & 14,3 & 11,6 & 10,8 & 13,6 & 11,9 & 11,7 \\
\hline \multirow[t]{5}{*}{ Eiweiß } & 0. & 16,9 & 23,9 & 19,0 & 18,2 & 24,1 & 21,6 & 20,1 & 23,3 & 21,3 & 9,90 & 17,0 & 15,0 & 14,4 & 20,8 & 17,9 & 19,0 \\
\hline & 1. & 5,30 & 14,6 & 8,90 & 9,10 & 14,3 & 12,4 & 7,30 & 16,6 & 11,1 & 4,60 & 7,75 & 6,90 & 6,30 & 12,3 & 9,90 & 9,80 \\
\hline & 2. & 5,30 & 8,40 & 6,20 & 4,50 & 10,8 & 8,90 & 3,50 & 6,05 & 4,90 & 4,20 & 8,88 & 5,60 & 3,45 & 12,1 & 6,10 & 6,30 \\
\hline & 3. & 3,90 & 6,70 & 5,00 & 3,30 & 5,90 & 4,90 & 3,40 & 4,75 & 4,20 & 3,10 & 9,80 & 6,10 & 2,98 & 12,4 & 6,00 & 5,20 \\
\hline & 4. & 3,80 & 7,40 & 5,10 & 4,40 & 5,05 & 4,70 & 4,90 & 4,90 & 4,10 & 3,30 & 4,70 & 4,00 & 3,88 & 5,45 & 4,60 & 4,50 \\
\hline \multirow[t]{5}{*}{ Fett } & 0. & 2,46 & 12,7 & 6,43 & 3,92 & 14,6 & 9,48 & 6,00 & 12,2 & 9,75 & 3,34 & 9,96 & 5,88 & 1,64 & 9,26 & 4,99 & 7,30 \\
\hline & 1. & 0,68 & 12,8 & 3,95 & 2,62 & 4,26 & 3,40 & 1,70 & 3,60 & 2,80 & 1,66 & 3,18 & 2,05 & 0,72 & 2,20 & 1,45 & 2,62 \\
\hline & 2. & 0,62 & 5,20 & 2,40 & 2,02 & 5,66 & 3,66 & 1,24 & 4,54 & 2,48 & 0,66 & 6,68 & 2,32 & 0,78 & 2,72 & 1,57 & 2,49 \\
\hline & 3. & 1,32 & 4,80 & 3,31 & 0,68 & 7,00 & 4,14 & 1,64 & 10,5 & 4,83 & 1,04 & 6,36 & 3,67 & 0,80 & 3,32 & 1,76 & 3,54 \\
\hline & 4. & 1,06 & 3,54 & 2,39 & 0,56 & 3,20 & 1,87 & 1,44 & 5,52 & 2,75 & 0,66 & 6,78 & 2,71 & 2,16 & 5,58 & 3,46 & 2,64 \\
\hline \multirow[t]{5}{*}{ Zucker } & 0. & 0,98 & 4,16 & 2,69 & 0,50 & 1,54 & 1,12 & 0,74 & 1,62 & 1,15 & 0,57 & 2,00 & 1,26 & 1,04 & 1,76 & 1,42 & 1,53 \\
\hline & 1. & 2,14 & 4,70 & 3,50 & 0,24 & 2,44 & 1,49 & 0,70 & 2,62 & 1,87 & 1,24 & 2,70 & 2,12 & 1,48 & 2,56 & 2,15 & 2,23 \\
\hline & 2. & 2,18 & 4,96 & 3,35 & 0,46 & 3,72 & 2,16 & 2,48 & 4,12 & 3,32 & 0,80 & 3,08 & 2,22 & 1,08 & 3,00 & 2,14 & 2,64 \\
\hline & 3. & 1,26 & 3,12 & 2,52 & 2,62 & 4,08 & 3,45 & 3,14 & 4,34 & 3,80 & 0,78 & 2,84 & 2,14 & 1,04 & 3,42 & 2,27 & 2,84 \\
\hline & 4. & 1,06 & 3,26 & 2,52 & 3,06 & 4,98 & 3,93 & 3,94 & 4,20 & 4,04 & 1,76 & 3,42 & 2,73 & 2,10 & 3,52 & 2,77 & 3,20 \\
\hline \multirow[t]{5}{*}{ Asche } & 0. & 0,83 & 1,24 & 1,09 & 1,02 & 1,47 & 1,18 & 1,14 & 1,33 & 1,25 & 1,07 & 1,22 & 1,13 & 1,07 & 1,30 & 1,17 & 1,16 \\
\hline & 1. & 0,72 & 0,93 & 0,85 & 0,98 & 1,18 & 1,09 & 0,87 & 1,46 & 1,12 & 0,89 & 1,08 & 0,99 & 0,86 & 1,09 & 1,00 & 1,01 \\
\hline & 2. & 0,78 & 0,98 & 0,86 & 0,82 & 1,22 & 0,99 & 0,80 & 0,94 & 0,87 & 0,84 & 1,08 & 0,93 & 0,86 & 1,06 & 0,95 & 0,92 \\
\hline & 3. & 0,76 & 0,99 & 0,87 & 0,81 & 1,07 & 0,90 & 0,77 & 0,89 & 0,82 & 0,88 & 1,13 & 1,00 & 0,91 & 1,08 & 0,95 & 0,91 \\
\hline & 4. & 0,80 & 0,94 & 0,87 & 0,81 & 1,28 & 0,95 & 0,75 & 0,98 & 0,87 & 0,84 & 1,05 & 0,97 & 0,78 & 1,01 & 0,88 & 0,91 \\
\hline
\end{tabular}

den von obigen Autoren untersuchten Milchrasssen (außer Schwarze Angus) Die Eiweißwerte beim Ungarischen Fleckvieh - als Fleischrasse gehalten - ähneln mehr den Milchrassen mit Jersey-Vätern.

Der von ÁLDÁSY und ERỔS (1969) festgestellte Gehalt an Gesamteiweiß im Kolostrum des Ungarischen Fleckviehs - unmittelbar nach dem Kalben gemessen - stimmt mit den in vorliegenden Untersuchungen erhaltenen Ergebnissen überein. Genannte Autoren erhielten für 26 Kühe durchschnittlich 14,2\% (min. 9,6\%, max. 19,6\%). Nach 24 Stunden waren die gemessenen Werte etwas höher als die vorliegenden: 7,5\% (min. $5,8 \%$, max. 10,8\%), nach 72 Stunden jedoch bedeutend niedriger: $2,4 \%$ (min. $1,2 \%$, max. 3,7\%) und dies nicht nur beim Ungarischen Fleckvieh. Der von KVAPILIK et al. (1975) untersuchte Eiweißgehalt bei Milchkühen stimmt - mit Ausnahme der 14,64\% vom ersten Tag - mit den erhaltenen, zum ähnlichen Zeitpunkt festgestellten Durchschnittswerten bei den Fleischrassen überein (3.Tag: 4,59\%, 5.Tag: 4,16\%, 5.Tag: 4,16\%), obwohl der Eiweißwert des 5. Tages in vorliegender Untersuchung nur geschätzt wurde. Auch bei diesen Merkmalen sind die Werte des hier untersuchten Ungarischen Fleckviehs den Werten genannter Autoren am ähnlichsten. Anders sind die Ergebnisse bei Kühen des Dänischen Rotviehs, die von SEBELA und KLICNIK 
(1975) untersucht wurden. Bei ihnen ist der Eiweißgehalt im Kolostrum des 1. Tages bedeutend niedriger als bei allen hier untersuchten Rassen (10,38\% [min. 3,55\%, max. $15,18 \%])$ oder anderen im Schriftum untersuchten Milchrassen $(\mathrm{P} \leq 0,1)$. Das trifft auch für den 3 . Tag $(-3,88 \%)$ bzw. den 5 . Tag $(-3,29 \%) \mathrm{zu}$.

Hinsichtlich des Trockensubstanzgehaltes war am 2. Tag bei fast allen Rassen ein Bruch zu erkennen, wofür eindeutig das Absinken des Fettgehaltes im Kolostrum verantwortlich ist (Korrelationskoeffizient der 125 Proben: $r=0,80$ ). Dieser drastische Rückgang ist bereits am Tag nach der Abkalbung zu beobachten, was im Falle der Fleischrasse vielleicht mit einer größeren Verdünnung zusammenhängt. Der Fettgehalt hat am 3. Tag nach der Abkalbung den zu erwartenden Wert.

Laut MERÉNYI et al. (1996) ist der Zuckergehalt in der Kolostralmilch niedriger als in der Vollmilch. Das wird durch vorliegende Ergebnisse bestätigt. In den Kolostrumproben stieg der Zuckergehalt von der Abkalbung bis zum 4. Tag ständig an und erreicht voraussichtlich am 5.-6. Tag den für Vollmich charakteristischen Wert von 4,8$5,0 \%$.

Der Aschegehalt im Kolostrum der hier untersuchten Fleischrassen, unmittelbar nach dem Abkalben gemessen, stimmt mit den Werten von KLIMMER und SCHÖNBERGH (1951), SEBELA und KLICNIK (1975) sowie CSAPÓ (1992) überein, liegt aber unter den Werten von KIRCHMEIER (1976). Nach KLIMMER und SCHÖNBERGH (1951) erreicht der Aschegehalt bereits am 3. Tag nach der Abkalbung den normalen Vollmilchwert, was vorliegende Ergebnisse aber nicht bestätigen. Obwohl die Komponenten des Kolostrums nur bis zum 4. Tag nach der Abkalbung untersucht wurden, stimmen wir mit den von SEBELA und KLICNIK (1975), KIRCHMEIER (1976) bzw. SEBELA und KLICNIK (1975) gemachten Feststellungen überein, wonach der Aschegehalt am 7.-10. Tag bzw. 13.-14. Tag der Laktation den Vollmilchwert erreicht. Da dieser Wert nach früheren Untersuchungen im Durchschnitt von 4 Fleischrassen $0,75 \%$ beträgt (KOVÁCS, 1997a), bedeuten die vorliegenden am 4 . Tag gemessenen $0,91 \%$ für das zeitliche Absinken des Aschegehalts ca. die Hälfte. Vorliegende Ergebnisse 24 Stunden nach der Abkalbung bestätigen die Werte von KIRCHMEIER (1976), wonach der Aschegehalt im Kolostrum bereits am Ende des 1. Tages auf 1,0\% sinkt. Ähnliche Ergebnisse zeigten auch die Untersuchungen von SEBELA und KLICNIK (1975) an Kühen des Dänischen Rotviehs für den am 2. Tag gemessenen Aschegehalt, der einen Durchschnittswert von 0,91\% hatte. Der Aschegehalt im Kolostrum der Rasse Limousin ist dem von CSAPÓ (1992) angegebenen Wert bei Kühen mit Jersey-Vätern am nächsten. Der Wert bei Kühen mit Holstein-Friesian-Vätern liegt bei ihm unter den hier errechneten Werten.

Untersuchung der Eiweißfraktionen im Kolostrum verschiedener Fleischrinderrassen in Abhängigkeit von der nach dem Abkalben vergangenen Zeit

In der Tabelle 4 sind die Veränderungen der wichtigsten Eiweißfraktionen aufgeführt in Abhängigkeit von der nach dem Abkalben vergangenen Zeit, gegliedert nach den untersuchten Fleischrinderrassen. 
Tabelle 4

Wichtigste Eiweißfraktionen im Kolostrum einiger Fleischrinderrassen in Abhängigkeit von der nach dem Abkalben vergangenen Zeit (Examination of the protein fractions at some beef cattle breeds' colostrum in different time after calving )

\begin{tabular}{|c|c|c|c|c|c|c|c|c|c|c|c|c|c|c|c|c|c|}
\hline \multirow{2}{*}{$\begin{array}{l}\text { Kompo- } \\
\text { nente }\end{array}$} & \multirow[t]{2}{*}{ Tage } & \multicolumn{3}{|c|}{ Blonde d' Aq. } & \multicolumn{3}{|c|}{ Red Angus } & \multicolumn{3}{|c|}{ Schwarze Angus } & \multicolumn{3}{|c|}{ Ung. Fleckvieh } & \multicolumn{3}{|c|}{ Limousin } & \multirow{2}{*}{$\begin{array}{c}\text { Rassen } \\
\varnothing\end{array}$} \\
\hline & & $\min$ & $\max$ & $\varnothing$ & $\min$ & $\max$ & $\varnothing$ & $\min$ & & $\emptyset$ & & & $\varnothing$ & $\min$ & & $\varnothing$ & \\
\hline \multirow{5}{*}{$\begin{array}{l}\text { Molken- } \\
\text { eiweiß }\end{array}$} & 0. & 10,6 & 17,5 & 12,9 & 12,2 & 21,8 & 15,8 & 14,6 & 20,3 & 16,9 & 5,12 & 13,0 & 10,3 & 8,56 & 16,7 & 11,8 & 13,56 \\
\hline & 1. & 2,24 & 9,60 & 4,50 & 5,20 & 10,1 & 7,83 & 3,76 & 10,4 & 6,49 & 1,28 & 4,08 & 3,26 & 2,12 & 6,96 & 5,02 & 5,42 \\
\hline & 2. & 1,60 & 3,04 & 2,18 & 1,88 & 9,84 & 5,14 & 1,52 & 3,00 & 2,11 & 1,12 & 4,24 & 2,14 & 1,24 & 6,40 & 2,56 & 2,83 \\
\hline & 3. & 1,52 & 2,64 & 1,95 & 1,16 & 3,20 & 1,97 & 1,20 & 2,43 & 1,65 & 8 & 4,04 & 2,23 & 1,12 & 6,32 & 2,30 & 2,02 \\
\hline & 4. & 1,44 & 3,52 & 1,92 & 1,32 & 2,12 & 1,70 & 1,12 & 2,92 & 1,79 & 1,04 & 1,44 & 1,33 & 0,92 & 1,32 & 1,12 & 1,57 \\
\hline \multirow[t]{5}{*}{ Kasein } & 0. & 4,94 & 8,61 & 6,10 & 2,29 & 6,67 & 5,80 & 3,00 & & 4,30 & 3,65 & 6,66 & 4,63 & 4,08 & 7,40 & 6,03 & 5,37 \\
\hline & 1. & 2,90 & 5,94 & 4,38 & 3,22 & 6,02 & 4,53 & 44 & 6,20 & 4,57 & 1,28 & 6,47 & 3,63 & 4,18 & 6,46 & 4,90 & 4,40 \\
\hline & 2. & 3,20 & 36 & 4,00 & 2,62 & 4,52 & 3,78 & 1,38 & 3,48 & 2,81 & 2,68 & 4,64 & 3,44 & 1,45 & 5,70 & 3,53 & 3,51 \\
\hline & 3. & 1,82 & 4,06 & 3,03 & 2,70 & 3,94 & 3,49 & 1,88 & 3,31 & 2,56 & 1,42 & 5,76 & 3,83 & 1,62 & 6,07 & 3,66 & 3,31 \\
\hline & 4. & 2,36 & 3,88 & 3,14 & 2,80 & 3,28 & 3,05 & 1,72 & 3,33 & 2,35 & 1,58 & 3,66 & 2,67 & 2,88 & 4,13 & 3,45 & 2,93 \\
\hline \multirow{5}{*}{$\begin{array}{l}\text { NPN x } \\
6,38\end{array}$} & 0. & 0,30 & 0,53 & 0,44 & 0,24 & 0,60 & 0,39 & 0,30 & 1,53 & 0,68 & 0,39 & 0,75 & 0,54 & 0,33 & 0,55 & 0,44 & 0,50 \\
\hline & 1. & 0,27 & 0,53 & 0,35 & 0,18 & 0,64 & 0,34 & 0,21 & 0,60 & 0,34 & 0,33 & 0,69 & 0,48 & 0,27 & 0,57 & 0,36 & 0,37 \\
\hline & 2. & 0,17 & 0,42 & 0,31 & 0,24 & 0,61 & 0,40 & 0,27 & 0,94 & 0,50 & 0,25 & 0,71 & 0,46 & 0,18 & 1,22 & 0,48 & 0,43 \\
\hline & 3. & 0,24 & 0,74 & 0,47 & 0,18 & 0,86 & 0,46 & 0,18 & 0,45 & 0,33 & 0,21 & 0,94 & 0,48 & 0,22 & 0,55 & 0,36 & 0,42 \\
\hline & 4. & 0,24 & 0,97 & 0,49 & 0,31 & 0,69 & 0,51 & 0,24 & 0,56 & 0,43 & 0,20 & 0,95 & 0,45 & 0,15 & 0,35 & 0,39 & 0,42 \\
\hline
\end{tabular}

Vergleicht man die Ergebnisse hinsichtlich des Molkeneiweißgehaltes im Kolostrum mit denen der Literatur, ist festzustellen, daß die Unterschiede zwischen den von CSAPÓ und CSAPÓNÉ (1984) sowie SZENTPÉTERI et al. (1986) untersuchten Milchkühen zugunsten der Fleischrassen signifikant sind, mit Ausnahme des Ungarischen Fleckviehs. Ein großer Unterschied besteht auch darin, daß bei den von den genannten Autoren untersuchten Milchkühen nach der Abkalbung der Molkeneiweißgehalt viel drastischer absinkt als bei den hier untersuchten Fleischrassen und schon am 5. Tag den Vollmilchwert mit 0,8-0,9\% erreicht. Dagegen ist bei den untersuchten Fleischrassen das Absinken geringer und erfolgt parallel zum Absinken des Gesamteiweißgehaltes (Tab. 5). KVAPILIK et al. (1975) stellt bei Milchkühen am ersten Tag einen Serumeiweißgehalt von $8,26 \%$ fest, der am 3. Tag auf $1,34 \%$ und am 5 .Tag auf $1,01 \%$ zurückfiel. Die Werte des 3. bzw. 5. Tages liegen etwas über den von CSAPÓ und CSAPÓNÉ (1984) sowie SZENTPÉTERI et al. (1986) gemessenen, sind aber immer noch niedriger als die hier zum ähnlichen Zeitpunkt ermittelten.

Hinsichtlich des Kaseingehaltes im Kolostrum gibt es zwischen den von CSAPÓ und CSAPÓNÉ (1984) sowie SZENTPÉTERI et al. (1986) untersuchten Milchkühen und den untersuchten Fleischkühen keinen so großen Unterschied wie beim Molkeneiweiß. Die Durchschnittswerte der nach dem Abkalben zu verschiedenen Zeiten genommenen Proben zeigen sogar große Ähnlichkeit. Der Kaseingehalt im Kolostrum der Blonde d'Aquitaine, Red Angus und Schwarze Angus - höherer Anfangswert, stärkeres Absinken - ähnelt am meisten den Werten der von den obigen Autoren untersuchten Milchkühen mit Holstein-Friesian-Vätern, während die Kaseinwerte beim Ungarischen Fleckvieh bzw. bei Limousin - niedrigerer Anfangswert, geringeres Absinken - den Milchkühen mit Jersey-Vätern näher steht. Der Kaseingehalt im Kolostrum den von KVAPILIK et al. (1975) untersuchten Milchkühen kommt dem der untersuchten 
Blonde d'Aquitaine am nächsten. Außer den Blonde d'Aquitaine hatte noch die Limousinrasse die höchsten Kaseinwerte.

Hinsichtlich des NPN-Gehaltes ergaben sich ähnliche Feststellungen wie beim Molkeneiweiß. Er lag unmittelbar nach dem Abkalben sowie 24 Stunden danach auf einem Niveau von $\mathrm{P} \leq 10 \%$, ab dem 2 . Tag dagegen mit $\mathrm{P} \leq 0,1 \%$ auch noch höher als bei den Milchrassen, die von CSAPÓ und CSAPÓNÉ (1984) sowie SZENTPÉTERI et al. (1986) untersucht wurden. Das bedeutet, daß, ähnlich wie beim Gehalt an Molkeneiweiß, das Absinken des NPN-Gehaltes im Kolostrum der Fleischrassen geringer ist.

Tabelle 5 zeigt die Eiweißfraktionen bzw. die Zusammenhänge zwischen den Eiweißfraktionen und dem Gesamteiweißgehalt. Die Matrix enthält die Durchschnittswerte des Untersuchungsintervalles.

Tabelle 5

Gesamteiweißgehalt im Kolostrum einiger Fleischrassen bzw. die Korrelationen zwischen Eiweißfraktionen im Durchschnitt der ersten 4 Tage nach dem Abkalben (Correlation among the total protein content and the protein fractions in average of the first four days)

\begin{tabular}{lcccc}
\hline Komponente & Gesamteiweiß & Molkeneiweiß & Kasein & NPN x 6,38 \\
\hline Gesamteiweiß & 0,984 & & & \\
Molkeneiweiß & 0,630 & 0,520 & & \\
Kasein & 0,066 & 0,121 & $-0,179$ & \\
NPN x 6,38 & & & \\
\hline
\end{tabular}

Aus der Tabelle ist zu entnehmen, daß der einzige enge und positive Zusammenhang der untersuchten Proben zwischen dem Gehalt an Gesamteiweiß und Molkeneiweiß besteht. Veränderungen im Gesamteiweißgehalt und Veränderungen im Molkeneiweißgehalt haben demnach in Abhängigkeit von der Zeit nach dem Abkalben eine sehr ähnliche Tendenz. Besonders in der Zeit vom 0.-3. Tag. Auch die Korrelation zwischen dem Gesamteiweißgehalt und dem Kaseingehalt ist uberraschend groß, und sie vergrößert sich mit wachsendem Zeitabstand nach dem Kalben. Obwohl sich das Molkeneiweiß-Kasein-Verhältnis beim Übergang von der Kolostralmich zur Vollmilch umkehrt, verringert sich sein prozentualer Anteil, ähnlich der anderer Fraktionen, in jedem Fall mit wachsendem Zeitabstand nach dem Abkalben. Das bestätigen auch MERÉNYI et al. (1996), die neben dem hohen Molkeneiweißgehalt auch mehr Kasein in der Kolostralmilch feststellten. Die Autoren bemerken dazu, daß dies besonders wichtig ist, da dies die Haupteiweißquelle für das Kalb darstellt. Der hohe Molkeneiweißgehalt ist deshalb bedeutungsvoll, weil der biologische Wert des Molkeneiweißes fast das 1 1/2-fache des biologischen Wertes des Kaseins beträgt (CSAPÓ et al., 1982) und weil diese Fraktion auch die Immunglobuline enthält (CSAPÓ et al., 1991, 1994). In der Fleischrinderhaltung ist ein hoher biologischer Wert der Muttermilch besonders wichtig, weil sie die ausschließliche Nahrung des Kalbes ist (KOVÁCS, 1997b).

Aus der Tabelle 6 ist das Molkeneiweiß-Kasein-Verhältnis bei den verschiedenen Probeentnahmezeitpunkten, untergliedert nach Rassen, zu entnehmen.

Es ist ersichtlich, daß fast unabhängig von der Zeit nach dem Abkalben, beide Angusrassen im Molkeneiweiß-Kasein-Verhältnis von den anderen Fleischrassen zugunsten des Anteils an Molkeneiweiß abweichen. Der Kaseinanteil dagegen ist wiederum ge- 
genüber den Limousin bzw. den Blonde d'Aquitaine bedeutend.

Tabelle 6

Verhältnis von Molkeneiweiß:Kasein im Kolostrum einiger Fleischrinderrassen (Ratio of the whey protein and the casein of some beef cattle breeds' colostrum in different time after calving)

\begin{tabular}{lccccc}
\hline \multirow{2}{*}{ Rasse } & \multicolumn{5}{c}{ Molkeneiweiß-Kasein-Verhältnis (\%) } \\
0. Tag & 1. Tag & 2. Tag & 3. Tag & 4. Tag \\
\hline Blonde d'Aquitaine & $67,9: 32,1$ & $50,7: 49,3$ & $35,3: 64,7$ & $39,2: 60,8$ & $37,9: 62,1$ \\
Red Angus & $73,2: 26,8$ & $63,4: 36,6$ & $57,6: 42,4$ & $36,1: 63,9$ & $35,8: 64,2$ \\
Schwarze Angus & $79,7: 20,3$ & $58,7: 41,3$ & $42,9: 57,1$ & $39,2: 60,8$ & $43,2: 56,8$ \\
Ungarisches Fleckvieh & $69,0: 31,0$ & $47,3: 52,7$ & $38,3: 61,7$ & $36,8: 63,2$ & $33,3: 66,7$ \\
Limousin & $66,2: 33,8$ & $50,6: 49,4$ & $42,0: 58,0$ & $39,6: 61,4$ & $24,5: 75,5$ \\
Durchschnitt & $71,6: 28,4$ & $55,2: 44,8$ & $44,6: 55,4$ & $37,9: 62,1$ & $34,9: 65,1$ \\
\hline
\end{tabular}

Der Molkeneiweißgehalt im Kolostrum sowie die daraus errechneten ImmunglobulinG-Werte

Da es bei Rindern keine diaplazentare Immmunstoffübertragung gibt, erfolgt diese nach der Geburt über das Kolostrum. Die Immunstoffe werden im Darm absorbiert, gelangen so in die Blutbahn und entfalten dort ihre schützende Tätigkeit. Deshalb liegt die einzige Möglichkeit der natürlichen Immunisierung im Immunglobulingehalt des Kolostrums (EHRLICH, 1892).

Tabelle 7 zeigt die Durchschnittswerte des Molkeneiweißgehaltes sowie die daraus errechneten IgG-Werte.

Tabelle 7

Durchschnittlicher Molkeneiweißgehalt und die daraus errechneten Immunglobulin-G-Werte (Average of the whey protein of colostrum and the derivated values of Immunglobulin-G)

\begin{tabular}{|c|c|c|c|c|c|c|}
\hline & $\begin{array}{c}\text { Blonde } \\
\text { d'Aquitaine }\end{array}$ & $\begin{array}{l}\text { Red } \\
\text { Angus }\end{array}$ & $\begin{array}{c}\text { Rasse } \\
\text { Schwarze } \\
\text { Angus }\end{array}$ & $\begin{array}{l}\text { Ungarisches } \\
\text { Fleckvieh }\end{array}$ & Limousin & $\begin{array}{l}\text { Durch- } \\
\text { schnitt }\end{array}$ \\
\hline \multicolumn{7}{|c|}{ Molkeneiweiß (\%) } \\
\hline 0 . Tag & 12,86 & 15,83 & 16,92 & 10,33 & 11,84 & 13,56 \\
\hline 1. Tag & 4,50 & 7,83 & 6,49 & 3,26 & 5,02 & 5,42 \\
\hline 2. Tag & 2,18 & 5,14 & 2,11 & 2,14 & 2,56 & 2,83 \\
\hline 3. Tag & 1,95 & 1,97 & 1,65 & 2,23 & 2,30 & 2,02 \\
\hline 4. Tag & 1,92 & 1,70 & 1,79 & 1,33 & 1,12 & 1,57 \\
\hline \multicolumn{7}{|c|}{$\begin{array}{l}\text { Immunglobulin-G } \\
(\mathrm{mg} / \mathrm{ml})\end{array}$} \\
\hline 0. Tag & 129,13 & 159,04 & 170,01 & 103,65 & 118,86 & 136,18 \\
\hline 1. Tag & 44,94 & 78,48 & 64,98 & 32,46 & 50,18 & 54,21 \\
\hline 2. Tag & 21,58 & $\$ 1,39$ & 20,88 & 21,18 & 25,41 & 28,13 \\
\hline 3. Tag & 19,27 & 19,47 & 16,24 & 22,09 & 22,79 & 19,97 \\
\hline 4. Tag & 18,96 & 16,75 & 17,65 & 13,02 & 10,91 & 15,44 \\
\hline
\end{tabular}

Vergleicht man die hier errechneten Werte mit denen aus der Literatur, so ist festzustellen, daß der Immunglobulin-G-Gehalt im erstgemolkenen Kolostrum der von ÁLDÁSY und ERÕS (1969) untersuchten Ungarischen Fleckviehkühe sowie den von MULLER und ELLINGER (1981) untersuchten Genotypen Ayrshire, Schweizer Braune, Guernsey, Holstein-Friesian und Jersey wesentlich geringer ist als bei den hier 
untersuchten Fleischrassen, bei denen der Immunglobulin-G-Gehalt zu ähnlichen Zeitpunkten gemessen wurde. Allerdings gibt es kaum Abweichungen zwischen den hier untersuchten Limousin und den von NORMAN und HOHENBOKEN (1981) untersuchten Simmentalern sowie den von CSAPÓ und CSAPÓNÉ (1984) untersuchten Milchkühen mit Holstein-Friesian-Vätern, wobei der Immunglobulin-G-Gehalt im erstgemolkenen Kolostrum 120,20 mg/ml beträgt. Der hier errechnete Wert für Ungarische Fleckviehkühe von $102,41 \mathrm{mg} / \mathrm{ml}$ zeigt wiederum Ähnlichkeit mit dem von den letztgenannten Autoren untersuchten Milchkühen mit Jersey-Vätern.

Der zeitliche Verlauf der Kurve des IgG-Gehaltes ist dagegen völlig anders als der in der Literatur beschriebene. ÁLDÁSY und ERÔS (1969) stellten bei Ungarischen Fleckviehkühen und CSAPÓ und CSAPÓNÉ (1984) bei verschiedenen Milchrassen einen viel drastischeren Abfall des IgG-Wertes im Kolostrum fest, als in den hier untersuchten Fleischrassen. ÁLDÁSY und ERÖS (1969) ist zuzustimmen, wonach jeder Liter Kolostralmilch vom 1. Tag vom Immunwert aus gesehen 9-10 1 Kolostralmilch vom 3. Tag entspricht. Ebenfalls nach ÁLDÁSY und ERŐS (1969), PORTER (1972) sowie LOSONCZY et al. (1979), können die im Kolostrum enthaltenen Immunglobuline von Kälbern nur bis zur 25.-27. Lebensstunde aufgenommen werden und sinken ab dem 3. Tag drastisch ab. Vorliegende Ergebnisse bestätigen auch die Feststellung von MIELKE (1979), wonach der Immunglobulin-G-Gehalt im erstgemolkenen Kolostrum in erster Linie von der Rasse abhängig ist. Die hier vorgelegten Ergebnisse zeigen, daß Unterschiede mit wachsendem Zeitabstand von der Abkalbung immer bedeutungsloser werden.

\section{Energiegehalt im Kolostrum}

Das Kolostrum dient außer zur Versorgung des Kalbes mit Mineralstoffen, Vitaminen und Immunstoffen auch zur Versorgung mit Energie.

Tabelle 8 stellt die aus den Komponenten der Proben errechneten spezifischen biologischen Nutzwerte, die als Vergleich für den Energiegehalt des Kolostrums bei den untersuchten Fleischrassen dienen, dar.

Tabelle 8

Vergleich des Energiegehaltes des Kolostrums einiger Fleischrassen mit Hilfe der spezifischen biologischen Nutzwerte (Comparison of the energy content of some beef cattle breeds' colostrum using by specific physiological values)

\begin{tabular}{llllll}
\hline & \multicolumn{5}{c}{ Spezifische biologische Nutzwerte (MJ/kg) } \\
Rasse & 0. Tag & 1. Tag & 2. Tag & 3. Tag & 4. Tag \\
\hline Blonde d'Aquitaine & 6,309 & 3,666 & 2,573 & 2,579 & 2,238 \\
Red Angus & 7,590 & 3,707 & 3,323 & 3,045 & 2,218 \\
Schwarze Angus & 7,649 & 3,316 & 2,376 & 3,254 & 2,468 \\
Ungarisches Fleckvieh & 5,080 & 2,346 & 2,245 & 2,843 & 2,210 \\
Limousin & 5,258 & 2,632 & 2,025 & 2,104 & 2,612 \\
Durchschnitt & 6,365 & 3,084 & 2,504 & 2,758 & 2,349 \\
\hline
\end{tabular}

Da zwischen dem biologischen Nutzwert und dem Fettgehalt im Kolostrum eine $95,4 \%$-ige positive Korrelation vorhanden ist, ist es nicht überraschend, daß beide 
Angusrassen den anderen Rassen überlegen sind. Der erwähnte enge Zusammenhang weist auf das Absinken des spezifischen biologischen Nutzwertes hin, auch der am 2. Tag eingetretene Bruch. In der Reihenfolge für den Energiegehalt nimmt die Rasse Blonde d'Aquitaine den dritten Platz ein, was aber mit dem hohen Gesamteiweißgehalt im Kolostrum zusammenhängt (der Korrelationswert zwischen dem Gesamteiweißgehalt und dem spezifischen biologischen Nutzwert beträgt 94,6\%). Auch bei den Limousin gleicht der Gesamteiweißgehalt den niedrigen Nutzwert aus, wobei diese hinsichtlich des Fettgehaltes noch unter dem Ungarischen Fleckvieh liegen.

Wahrscheinlich ist es kein Zufall, daß die beiden erwähnten großrahmigen französischen Fleischrinder, die eine überdurchschnittliche Wachstumsintensität aufweisen, außer einem entsprechenden Energiegehalt auch den Eiweißbedarf über das Säugen decken können.

\section{Diskussion}

Die wichtigsten Komponenten des Kolostrums in Abhängigkeit von der nach dem $\mathrm{Ab}$ kalben vergangenen Zeit

Hinsichtlich des Trockensubstanzgehaltes sind die untersuchten Fleischrinderrassen im allgemeinen den in der Literatur erwähnten Milchrassen überlegen. Besonders unmittelbar nach dem Abkalben und am 1. Tag danach. Ab dem 2. Tag kehrt sich die Situation um und der Trockensubstanzgehalt der Milchrassen ist höher. Einen Übergang zwischen den beiden Nutzungstypen stellt das Ungarische Fleckvieh, als Fleischrasse gehalten, dar, bei dem der Trockensubstanzgehalt mehr nach den Milchrassen tendiert. Aus dieser Tatsache kann man schließen, daß die Kolostralmilch zu Beginn eine höherer Konzentration hat, aber dafür eher die Vollmilchwerte erreicht.

Für die Unterschiede im Trockensubstanzgehalt ist in erster Linie der Gesamteiweißgehalt verantwortlich, da die Korrelation zwischen ihnen sehr eng ist. Somit hat der Eiweißgehalt eine ähnliche Tendenz wie der Trockensubstanzgehalt, allerdings mit dem Unterschied, daß bei den hier untersuchten Fleischrindern der Eiweißgehalt nicht nur in den ersten 48 Stunden, sondern auch noch am 2.-4. Tag nach dem Abkalben höher war als bei den Milchkühen. Auch hier ist das Ungarische Fleckvieh eine Ausnahme. Sowohl beim prozentualen Anteil des Eiweißgehaltes als auch was das Absinken betrifft, hat es mehr Ähnlichkeit mit den Milchrassen.

Der Fettgehalt im Kolostrum verringert sich bei allen Fleischrinderrassen bereits am ersten und zweiten Tag, was einen Bruch im Trockensubstanzgehalt verursacht, da auch hier eine enge Korrelation besteht. Dieses Ergebnis bei den Fleischrinderrassen hängt möglicherweise mit der größeren Verdünnung zusammen.

Nach vorliegenden Untersuchungen erhöht sich der Zuckergehalt im Kolostrum kontinuierlich von der Abkalbung an und erreicht erwartungsgemäß am 5.-6. Tag den Vollmilchwert von 4,8-5,0\%.

Der Aschegehalt im Kolostrum der untersuchten Fleischrinder stimmt mit den in der Literatur publizierten Werten für Milchrassen überein, d.h. der Aschegehalt wird ca. am 7.-8. Tag nach der Abkalbung den Vollmilchwert erreichen. 
Untersuchung der Eiweißfraktionen im Kolostrum verschiedener Fleischrinderrassen in Abhängigkeit von der nach dem Abkalben vergangenen Zeit

Sowohl hinsichtlich des Gesamteiweißgehaltes als auch des Molkeneiweißgehaltes im Kolostrum kann ein signifikanter Unterschied zugunsten der untersuchten Fleischrassen gegenüber den Milchrassen festgestellt werden. Das ist kein Zufall wenn man berücksichtigt, daß die Korrelation zwischen den beiden Eiweißarten fast 1,000 beträgt. Somit ist im Vergleich zu den Milchrassen auch hier ein langsameres Absinken, ebenso wie beim Gesamteiweißgehalt, zu beobachten. Nach vorliegenden Berechnungen erreicht der Molkeneiweißgehalt bei den Fleischrindern erst nach dem 5. Tag den Vollmilchwert.

Beim Kaseingehalt im Kolostrum gibt es zwischen den untersuchten Fleischrindern und den in der Literatur vorliegenden Milchrinderwerten keinen so großen Unterschied wie beim Molkeneiweiß. Bei den Blonde d'Aquitaine und der Limousinerasse ist allerdings der Kaseingehalt höher als bei den übrigen und auch den in der Literatur angegebenen Milchtypen. Das zeigt sich auch im Molkeneiweiß-Kasein-Verhältnis, hauptsächlich bei der Limousinrasse. Gleichzeitig weicht der Anteil des Molkeneiweiß-Kasein-Verhältnisses beider Angusrassen zugunsten des Molkeneiweißanteils von dem der anderen Fleischrassen ab. Der hohe Anteil an Molkeneiweiß ist für die Widerstandsfähigkeit der Kälber wichtig.

Der NPN-Gehalt im Kolostrum hat einen ähnlichen Verlauf wie das Molkeneiweiß, d.h. daß auch diese Eiweißfraktion in größerer Menge in der Kolstralmilch der untersuchten Fleischrinder zu finden ist. Das Absínken des NPN-Gehaltes erfolgt noch langsamer als dies beim Molkeneiweißgehalt der Fall ist.

Der.Molkeneiweißgehalt im Kolostrum sowie die daraus errechneten.ImmunglobulinG-Werte

Die erechneten Immunglobulin-G-Werte im erstgemolkenen Kolostrum der Fleischkühe liegen höher als die in der Literatur gefundenen Daten bei Milchkühen. Nur Limousin und das Ungarische Fleckvieh sind Ausnahmen, deren Werte mit den im Schriftum beschriebenen Milchrassen übereinstimmen. Am 1.-4. Tag liegen die IgGWerte beider Rassen über denen der Milchrassen. Das hängt damit zusammen, daß die Verlaufskurve für den IgG-Gehalt bei den untersuchten Fleischrassen völlig von der der Milchrassen abweicht. Der Rückgang bei den Milchrassen ist viel drastischer als bei den hier erhaltenen Untersuchungsergebnissen und hat somit eine ähnlich sinkende Tendenz wie das Gesamt- und das Molkeneiweiß.

Die hier gefundenen Ergebnisse bestätigen die in der Literatur getroffene Feststellung, wonach der IgG-Gehalt im erstgemolkenen Kolostrum in erster Linie von der Rasse abhängig ist. Es konnte nachgewiesen werden, daß diese Unterschiede mit wachsendem Zeitabstand nach dem Abkalben an Bedeutung verlieren.

\section{Energiegehalt des Kolostrums}

Es wurde der spezifische biologische Nutzwert errechnet und als Ausdruck des Energiegehaltes des Kolostrums für den Vergleich der Fleischrinderrassen genutzt. Infolge 
der engen positiven Korrelation zwischen dem Energiegehalt des Kolostrums und dem Fettgehalt, verfügen beide Angusrassen über einen höheren Energiewert im Kolostrum als die übrigen Fleischrinder. Obgleich der spezifische biologische Nutzwert von Eiweiß nicht einmal die Hälfte des spezifischen biologischen Nutzwertes von Fett erreicht, nimmt die Rasse Blonde d'Aquitaine wegen ihres außerordentlich hohen Eiweißgehaltes die 3. Stelle hinsichtlich des Energiegehaltes im Kolostrum ein. Im Hintergrund steht der enge korrelative Zusammenhang zwischen dem Gesamteiweißgehalt und dem spezifischen biologischen Nutzwert. Auch bei den Limousin wird durch den Gesamteiweißgehalt im Kolostrum der niedrige Energiegehalt ausgeglichen. Bei den Ungarischen Fleckviehkühen erfolgt der Ausgleich durch den höheren Fettgehalt im Kolostrum. Es ist kein Zufall, daß die beiden erwähnten großrahmigen französischen Fleischrassen über eine überdurchschnittlich große Wachstumsintensität verfügen, denn neben einem entsprechend hohen Energiegehalt steht ihnen auch Eiweiß zur Verfügung. Das hat besonders auf den Muskelaufbau positiven Einfluß.

\section{Literatur}

ÁLDÁSY, P.; ERÖS, T.:

Importance of colostrum in rearing new-born calves healthy. (In Hungarian). Magyar Állatorvosok Lapja 24 (1969), 183-186

BÍRO, GY.; LINDNER, K.

Table of nutrition. (In Hungarian) Medicina Könyvkiadó Rt. Budapest, 16 p. (1995)

CSAPÓ, J.; HORVẢTHNÉ, A.M.; MAKAY, B.:

Comparison of the dry matter-, protein-, casein-, whey protein-, and non protein nitrogen content in colostrum and transition milk of Holstein, Hungarian Simental and Holstein x Hungarian Simental $F_{1}$ cows. (In Hungarian). Magyar Állatorvosok Lapja 6 (1982a), 411-414

CSAPÓ, J.; TERLAKY, B.É.; CSAPÓ, J.-ne; MAKAY, B.:

The amino acid composition and the change of that in colostrum and the transition milk of Holstein, Hungarian Simental and Holstein $x$ Hungarian Simental $F_{1}$ cows, after calving. (In Hungarian). Magyar Állatorvosok Lapja 6 (1982b), 415-419

CSAPÓ, J.; CSAPÓ, J.-ne:

Influence of Jersey $\mathrm{x}$ Holstein criss-crossing for the composition of colostrum and milk on the basis of „Hungarofríz“. (In Hungarian). Szaktanácsok (1984) 1, 32-37

CSAPÓ, J.; CSAPÓ-KISS, Zs.:

Biological value and change of milk protein in cattle, goats and sheep during lactation. Acta Alimentaria (1988), 4, $372 \mathrm{p}$.

CSAPÓ, J.; WOLF, Gy.; CSAPÓ-KISS, Zs.; SZENTPÉTERI, J.; KISS, J.: CSAPÓ, J.: Composition of colostrum from twinning cattle. Acta Agronomica Hungarica (1991) 1-2, 152-157

Composition of colostrum and milk of ruminants in Hungary, and analitics of some components). (In EHRLICH, P.: Hungarian). Akadémiai doktori értekezés. MTA, Budapest, 1992

KOVÁCS, A.Z.:

Über Immunität durch Vererbung und Zeugung. Z. Hyg. Infektionskrankheiten, 1892, 12, 183 p.

Composition of milk of some beef cattle breeds in Hungary. (In Hungarian). Allattenyéstes és Takarmányozás 46 (1997a) 2, 175-187

KOVÁCS, A.Z.:

The milking ability of angus cows in comparision with their calves growth. (In Hungarian). Acta Agraria Kaposváriensis, Kaposvár, 1997b

KIRCHMEIER, O.:

Eigenschaften der Milch. In: Handbuch der Lebensmittelchemie. Springer-Verlag Berlin - Heidelberg New York, 1976 
KLIMMER, M.; SCHÖNBERG, F.:

Milchkunde und Milchhygiene. 6. Auflage, Verlag M.H. Schaper, Hannover, 1951

KVAPILIK, J.; SUCHÁNEK, B.; BRAUNER, J.: Premena mleziva na mlékozralé se zamerenim na jeho chemické a technologické vlastnosti. Zivocisna Vyroba 20 (1975), 169-182

LOSONCZY, S.; PETHES, Gy.; FRENYO, V.L.; ANTAL, T.; SZABÓ, I.:

Fast physiological method to check calves in the consumption of colostrum under large-scale management. Changing of concentration of immunglobulin level in the pre- and postcolostral serum. (In Hungarian). Magyar Állatorvosok Lapja 34 (1979), 371-375

MERÉNYI, I.; LENGYEL, Z.:

MIELKE, H.: Handbook of milkfarming. (In Hungarian). Budapest 1996, 88 p.

Theoretische und praktische Aspekte der Ausbildung und Bewertung der kolostralen Immunität sowie Möglichkeiten einer gezielten Immunprophylaxe beim Kalb. Immunologie 34 (1979), 223-229

MULLER, L.D.; ELLINGER, D.K.:

Colostral immunglobulin concentrations among breeds of dairy cattle, J. Dairy Sci., Champaign, Ill. 64 (1981), 1727-1730

NORMAN, L.M.; HOHENBOKEN, W.D.:

Genetic differences in concentration of IgG and IgM in serum and colostrum of cows and in serum of neonatal calves. J. Animal Sci., Albany, N.Y. 53 (1981) 6, 1465-1472

PORTER, P.:

Immunglobulins in bovine mammary secretions. Quantitative changes in early lactation and absorption by the neonatal calf. Immunology 23 (1972), 225

SARUDI, I.:

Persönliche Mitteilung, 1998

SEBELA, F.; KLICNIK, V.:

Vlasnosti kravského mleziva. Acta univ. agr. (Brno) fac. Agron., 1975, 3, 517-525

SPSS for Windows

Version 7.5, copyright SPSS incorporation, 1996

SZENT-IVÁNYI, T.:

Immunological aspects of the embrios and the new-borns at farm animals. (In Hungarian). Magyar Állatorvosok Lapja 31 (1976), 619-622

SZENTPÉTERI, J. KARLE, G.; GUNDEL, J.ne.; CSAPÓ, J.; CSAPÓ, J.-ne:

Influence of crossing on the base of "Hungarofriz" by Jersey and Holstein to the composition of colostrum and milk. (In Hungarian). Állattenyéstes és Takarmányozás 6 (1986), 549-558

Eingegangen: 05.06.1998

Akzeptiert: 06.01.1999

Anschrin der Verfasser

Prof. Dr. JÁNOS IVÁNCSICS, Agraringenieur ATTILA Z. KOVÁCS

PANNON Agrarwissenschaftliche Universităt Mosonmagyaróvár

Lehrstuhl für Tierzucht

9201 Mosonmagyaróvár

Ungarn 